\title{
Decimetric Fine Structures as a Possible Signature of Chromospheric Evaporation
}

\author{
F. C. R. Fernandes, J. R. Cecatto, M. C. Andrade, F. R. H. Madsen, L. C. P. Moraes, and H. S. Sawant \\ Instituto Nacional de Pesquisas Espaciais, C.P. 515 12201-970, São José dos Campos, Brazil
}

Received on 6 February, 2004; revised version received on 26 April, 2004

\begin{abstract}
In this work, we concentrate in the analysis of radio slowly drifting fine structures associated with solar flares recorded in the frequency range of (1000 - 2500) MHz by the Brazilian Solar Spectroscope (BSS), in regular operation at National Institute of Space Research (INPE) - Brazil. The main morphological aspects of each fine structure are narrow-band of about $5-10 \mathrm{MHz}$ and small duration of the order of 50 milliseconds. The majority of these fine structures are observed over a time interval of a couple of minutes before the maximum of the associated flare, in the impulsive phase. However, some observations during the gradual decay phase are also reported. They drift towards lower frequencies, with slow rates typically of about of $10-100 \mathrm{MHz} \mathrm{s}^{-1}$. Estimated velocities of the exciter from the negatively drifting structures suggest that the shock-like exciter is propagating in the higher chromosphere. Than, those fine structures are interpreted as a possible signature in decimetric emissions from the chromospheric evaporation phenomenon. Details of these observations and their interpretation in terms of the plasma emission produced by accelerated particles and the chromospheric evaporation front is presented.
\end{abstract}

\section{Introduction}

Decimetric band is known to exhibit various types of fine structures [1-4]. However, observations in the decimetric band became stagnant till 80's. Around that time the Skylab satellite observations showed that energy to the flare is released where the electron density range is around $10^{9}$ $-10^{10} \mathrm{~cm}^{-3}$, i.e., in the decimetric range of the solar radio spectrum [5 and references within]. Since then, various high frequency-time resolution spectroscopes were put into operation [6]. The Brazilian Solar Spectroscope (BSS) [7, 8] belongs to this new generation of solar radio spectroscopes. BSS is in regular operation since 1998 and it has recorded many different types of fine structures. Here, we analyze some particular groups of decimentric fine structures with slowly drifting high frequency edge. Karlický et al. [9] also reported the observations of slowly drifting structures in decimetric range.

Recently, the chromospheric evaporation has been invoked to explain the occurrence of the slowly drifting fine structures in radio wavelengths [10]. In fact, the first suggestion the chromospheric evaporation could be investigated by radio observations in decimetric wavelengths generated by electron beam going down in magnetic loop and crossing up-going evaporation front was given by [1].

The basic scenario of chromospheric evaporation is the following $[12,13]$. During the very beginning of the flare, the turbulence in plasma of the active region begins and the flare energy is released up in the corona. This energy then accelerates particles and heats the plasma. The accelerated particles then move down toward the upper chromosphere along the magnetic lines, where they lose their energy by collisions with denser plasma. The heated plasma expands rapidly. However, it is confined by the magnetic loop struc- ture, and then it flows upward with velocities of about a few hundreds of $\mathrm{km} \mathrm{s}^{-1}$. The up-flow of the hot plasma is known as chromospheric evaporation [14]. The up-flowing hot plasma can be inferred by broadening and blue shifting of the lines as observed in soft X-ray [12, 13, 15-18].

Based especially on X-ray lines observations, many estimations of the velocity of chromospheric evaporation have been done. The determined evaporation front velocity ranges from aproximately $100 \mathrm{~km} \mathrm{~s}^{-1}$, for the transition region [19] or of about $150 \mathrm{~km} \mathrm{~s}^{-1}[19,20]$ for coronal lines up to $950 \mathrm{~km} \mathrm{~s}^{-1}$ for decimetric layer [10]. Also, Mariska et al. [21] estimated the velocity of $650 \mathrm{~km} \mathrm{~s}^{-1}$ and Silva et al. [13] inferred the interval of $350-450 \mathrm{~km} \mathrm{~s}^{-1}$ for the velocity of the evaporation front.

While the hot and dense plasma rises up it creates a discontinuity in temperature and density, in the loop. Beam of energetic electrons moving towards the foot-points of the loop interacts with this slowly upward moving "shock front" where a optical thickness $(\tau)$ is reduced due to its temperature dependence $\left(\tau \propto n \times T^{-3 / 2}\right)$ allowing to escape of high frequency radio emission. As the beam travels further it encounters high densities and lower temperature which increases opacity and radio emission is absorbed. Thus, as the shock front moves up, the cut off in high frequency will be slowly decreased allowing to infer the velocity of shock front. Eventually, the shock front will come in equilibrium with loop plasma and enabling escape of radio emission. Based on this scenario, Aschwanden and Benz [11] have suggested that the decimetric fine structures with slow drifting variation can be observed as result of the chomospheric evaporation process. However, there is a lack of observations in radio wavelengths as an evidence of emission caused by the cromospheric evaporation. 
TABLE 1. Solar flares showing decimetric slowly drifting fine structures.

\begin{tabular}{|c|c|c|c|c|c|c|c|c|}
\hline \multirow[b]{2}{*}{$\begin{array}{c}\text { Event } \\
\text { No. }\end{array}$} & \multirow[b]{2}{*}{ Day } & \multirow[b]{2}{*}{$\begin{array}{l}\text { Time } \\
\text { (UT) }\end{array}$} & \multirow[b]{2}{*}{$\begin{array}{l}\text { Band } \\
(\mathrm{MHz})\end{array}$} & \multicolumn{5}{|c|}{ Associated activity } \\
\hline & & & & $\begin{array}{l}\text { Start } \\
\text { (UT) }\end{array}$ & $\begin{array}{l}\text { Max } \\
\text { (UT) }\end{array}$ & $\begin{array}{l}\text { End } \\
\text { (UT) }\end{array}$ & $\begin{array}{l}\text { Goes } \\
\text { Class }\end{array}$ & $\begin{array}{c}\text { Active } \\
\text { Region \# }\end{array}$ \\
\hline 1 & 28/JUN/99 & $12: 21$ & $1615-1260$ & $12: 13$ & $12: 17$ & $12: 25$ & C1.6 & 8611 \\
\hline 2 & 27/NOV/99 & 12:08 & $1460-1015$ & 12:05 & $12: 12$ & $12: 16$ & $\mathrm{X} 1.4$ & 8771 \\
\hline 3 & 06/JUN/00 & $15: 05$ & $1660-1265$ & $14: 48$ & $15: 25$ & $15: 40$ & $\mathrm{X} 2.3$ & 9026 \\
\hline 4 & 06/JUN/00 & $16: 26$ & $1460-1210$ & $14: 48$ & $15: 25$ & $15: 40$ & $\mathrm{X} 2.3$ & 9026 \\
\hline 5 & 06/JUN/00 & $16: 32$ & $1350-1235$ & $14: 48$ & $15: 25$ & $15: 40$ & $\mathrm{X} 2.3$ & 9026 \\
\hline 6 & 16/SEP/00 & $14: 21$ & $1675-1535$ & $14: 16$ & $14: 28$ & $14: 32$ & M3.3 & 9165 \\
\hline 7 & 21/SEP/00 & $18: 22$ & $1130-1010$ & - & - & - & $245-606 \mathrm{MHz}$ & \\
\hline 8 & $24 / \mathrm{NOV} / 00$ & 15:07 & $1970-1880$ & $14: 51$ & $15: 13$ & $15: 21$ & $\mathrm{X} 2.3$ & 9236 \\
\hline 9 & 05/SEP/01 & $14: 32$ & $2170-2125$ & $14: 25$ & $14: 32$ & $14: 34$ & M6.0 & 9601 \\
\hline 10 & 04/APR/02 & $15: 28$ & $1240-1000$ & $15: 24$ & $15: 32$ & $15: 38$ & M6.1 & - \\
\hline 11 & 11/JUL/02 & $14: 48$ & $1190-1150$ & $14: 44$ & $14: 51$ & $14: 57$ & M5.8 & 10030 \\
\hline
\end{tabular}

Here, we present the decimetric fine structures associated with the solar flares recorded by BSS as a possible signature in decimetric waves of the chromospheric evaporation process. In section 2 we briefly describe the BSS instrument and its main characteristics. In section 3 the observations and the main properties of the fine structures recorded are presented. The shock velocity determinations and results are presented in section 4 and 5, respectively. The summary and conclusions are presented in section 6 .

\section{Instrumentation}

The BSS was put into regular operation at INPE for systematic solar observations in 1998 [8, 22]. It operates in conjunction with a 9 meter diameter polar mounted parabolic antenna, over the frequency range of $(1000-2500) \mathrm{MHz}$, with high time $(10-50 \mathrm{~ms})$ and frequency $(3 \mathrm{MHz})$ resolutions. The data are recorded in up to 100 digital channels [23], with absolute timing accuracy of 3 milliseconds. Thus, the BSS system has the capability to detect fine structures with narrow bandwidth and short durations [24].

\section{Decimetric observations}

Since the BSS has been put into regular operation, in 1998, it recorded more than 350 groups of solar decimetric emissions, most of them showing fine structures in time and/or frequency [25-28]. Out of them, we have selected the emissions associated with 8 solar flares recorded by BSS showing decimetric fine structures with high frequency edge slowly drifting from high to low frequencies observed in the period of March, 1999 to July, 2002. The Figure 1 shows the dynamic spectra of the recorded fine structures selected for analysis.

In the Table 1 we present a list of these emissions. The associated soft X-ray flares (GOES satellite) were also included. For 6 flares the fine structures occur prior the maximum of the soft X-ray associated emission, in the impulsive phase. In 3 cases, they are associated with the gradual decay phase of the X-ray emission, and for 1 case they are observed during the peak time of the soft X-ray emission. Except for 1 flare (classified as C1.6), the soft X-ray flares reported by GOES satellite associated with the fine structures were very intense: 3 flares reached $\mathrm{X}$ class and others 4 the M class.

\section{Data analysis}

\subsection{Frequency Drift Rate}

The high frequency and time measurements for each individual fine structure have been done using the BSSView and BSSData software. Then, the drift rate of the high frequency edge of each group of fine structures was determined from a linear regression in the frequency-time plane as shown in the Figure 2. The drift rates determined are given in the Table 2.

\subsection{Chromospheric Evaporation Shock Velo- city}

The frequency drift rate $(d f / d t)$ of the bursts group can be given by

$$
\frac{d f}{d t}=\frac{d f}{d N e} \frac{d N e}{d h} \frac{d h}{d t}=\frac{d f}{d N e} \frac{d N e}{d h} v_{c e} \cos \theta,
$$

where $f$ is the plasma frequency, $N_{e}$ is the electron density, $h$ is the height above the photosphere, $\theta$ is the angle between the direction of propagation of the front and the vertical direction and $v_{c e}$ is the chromospheric evaporation front velocity.

For the plasma emission the plasma frequency can be given as function of electron density in the solar atmosphere as:

$$
\left.f=s \times 8.98 \times 10^{-3} N_{e}^{1 / 2} \text { (in } \mathrm{MHz}\right) .
$$

where $s$ is the harmonic radiation number $(s=1$ and $s=2$ for fundamental and second harmonic emission, respectively). 


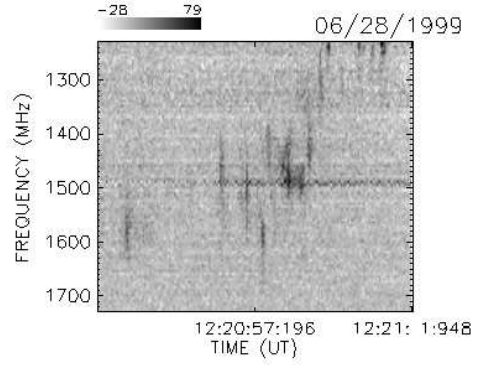

(a)

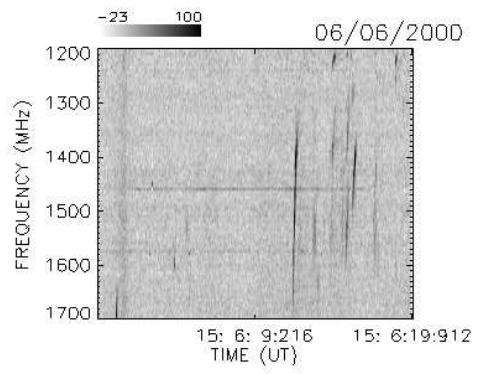

(c)

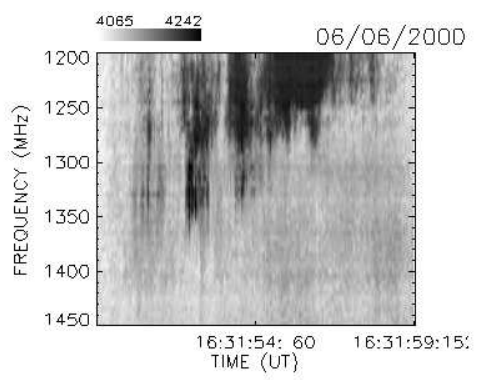

(e)

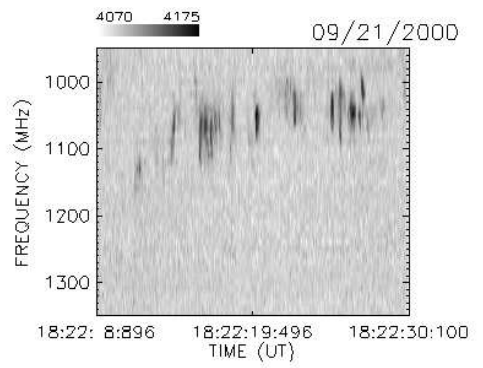

(g)

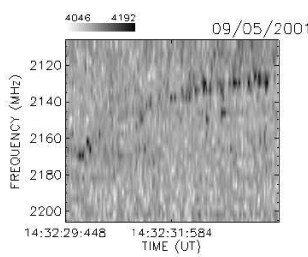

(i)

(j)

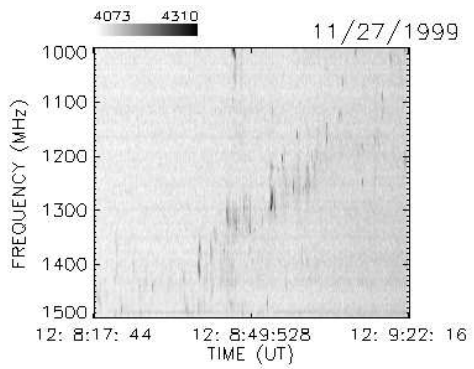

(b)

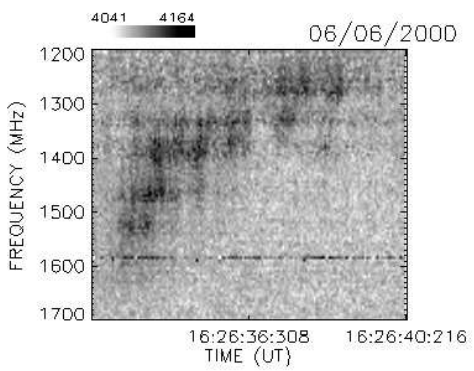

(d)

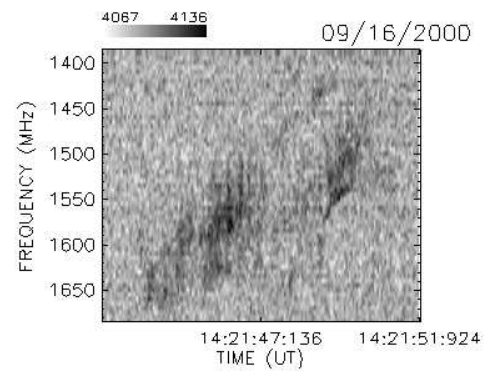

(f)

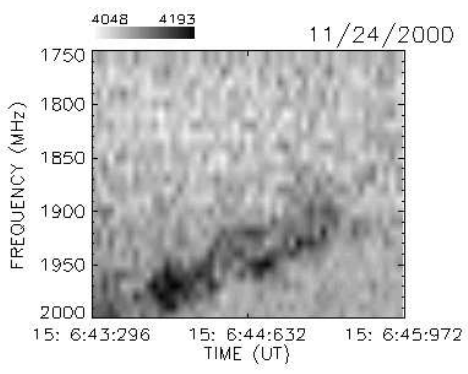

(h)

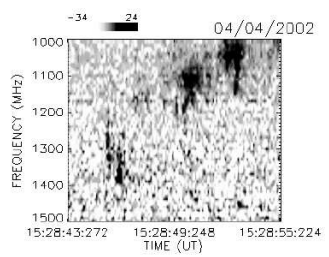

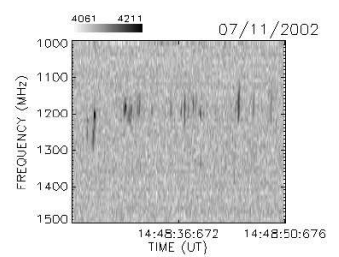

(k)

Figure 1. Examples of dynamic spectrum of decimetric fine structures showing slow drifting frequency variation: (a) June 28, 1999 ( $~ 12: 21$ UT); (b) November 27, 1999 ( 12:08 UT); (c) June 6, 2000 ( 15:05 UT); (d) June 6, 2000 ( 16:26 UT); (e) June 6, 2000 ( 16:32 UT); (f) September 16, 2000 ( 14:21 UT); (g) September 21, 2000 ( 18:22 UT); (h) November 24, 2000 ( 15:07 UT); (i) September 5, 2001 ( 14:32 UT); (j) April 4, 2002 ( 15:28 UT); (k) July 11, 2002 ( 14:48 UT). For all dynamic spectra, the background level of the emission was subtracted. 
TABLE 2. Measurements of the fine structures parameters.

\begin{tabular}{cccccc}
\hline $\begin{array}{c}\text { Event } \\
\text { No. }\end{array}$ & $\begin{array}{c}\Delta t \\
(\mathrm{~s})\end{array}$ & $\begin{array}{c}d f / d t \\
\left(\mathrm{MHz} \mathrm{s}^{-1}\right)\end{array}$ & $\begin{array}{c}\Delta N_{e} \\
\left(\times 10^{9} \mathrm{~cm}^{-3}\right)\end{array}$ & $\begin{array}{c}\Delta h \\
\left(\times 10^{3} \mathrm{~km}\right)\end{array}$ & $\begin{array}{c}v_{c e} \\
\left(\mathrm{~km} \mathrm{~s}^{-1}\right)\end{array}$ \\
\hline 1 & 4.5 & 80 & 3.1 & 7.7 & 1700 \\
2 & 30.0 & 15 & 2.3 & 9.0 & 300 \\
3 & 15.7 & 25 & 3.5 & 8.1 & 515 \\
4 & 2.5 & 100 & 1.0 & 3.2 & 1280 \\
5 & 6.0 & 19 & 1.0 & 3.1 & 520 \\
6 & 5.8 & 24 & 1.4 & 2.6 & 450 \\
7 & 15.3 & 8 & 0.8 & 3.9 & 250 \\
8 & 1.7 & 53 & 1.5 & 2.3 & 1350 \\
9 & 3.8 & 12 & 0.6 & 0.1 & 30 \\
10 & 7.2 & 38 & 1.7 & 7.4 & 1025 \\
11 & 23.0 & 2 & 0.3 & 1.7 & 75 \\
\hline
\end{tabular}

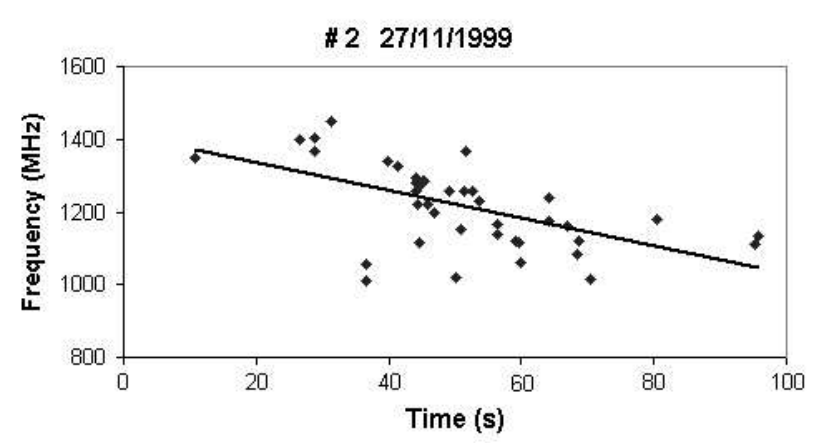

Figure 2. Example of frequency drift rate determination by linear fit of the high frequency edge points of the emission structures.

We have used a density model for the solar chromosphere derived from the statistical relation between drift rate and frequency of decimetric type III bursts [29]:

$$
\frac{d f}{d t}=A f^{\alpha}
$$

where $A=0.09$ and $\alpha=1.35$. The density model used [29], adapted from Aschwanden and Benz [11], assumes a powerlaw dependence for the low corona and an exponential shape for the upper corona as:

$$
N_{e}(h)=\left\{\begin{array}{ll}
N_{1}\left(\frac{h}{h_{1}}\right)^{-p} & \text { if } h \leq h_{1} \\
N_{q} \exp \left(\frac{-h}{H}\right) & \text { if } h>h_{1}
\end{array},\right.
$$

where $h$ is the height in the solar atmosphere, $h_{1}$ is the height of the transition in the density variation regime, where the power-law model is changed into exponential one, $N_{1}$ is the density transition, and $N_{q}$ is the electron density at the bottom of quiet solar corona obtained by barometric model, $p$ is the power-law index and $H$ is the density height scale. From the continuity conditions at the height $h_{1}$ we have:

$$
h_{1}=p H
$$

and

$$
N_{1}=N_{q} \exp (-p)
$$

As shown by Aschwanden and Benz [11], the constants $p$ and $N_{q}$ can be expressed as:

$$
p=\frac{2}{(\alpha-1)}
$$

and

$$
N_{q}=\left(\frac{e v}{2 A}\right)^{p}(s \times 8980)^{-2},
$$

where $v$ is the beam velocity expressed in units of the speed of light, $A$ and $\alpha$ are constants in the empirical type III drift rate [29]. Considering the second harmonic radiations $(s=$ 2) and the following values for the constants $H=7 \times 10^{4}$ $\mathrm{km}, h_{1}=3.5 \times 10^{5} \mathrm{~km}, N_{q}=4.6 \times 10^{8} \mathrm{~cm}^{-3}$ and $p=5$, we determined the density and height intervals covered by the fine structure groups.

\section{Results}

Considering the frequency drift rates for the high frequency edge of the decimetric emissions, the shock-front velocity of the source was estimated assuming a displacement in a vertical direction. The measurements of total duration of the global structure $(\Delta t)$, frequency drift rate $(d f / d t)$, density interval $\left(\Delta N_{e}\right)$, height interval $(\Delta h)$ and shock-front velocity of the fine structure source $\left(v_{c e}\right)$ are shown in Table 2.

\section{Summary and discussions}

We have presented some radio fine structures showing slowly drifting frequency associated with solar flares recorded Brazilian Solar Spectroscope (BSS), in the frequency range of (1000-2500) MHz. The common characteristics of those fine structures are the narrow-band and short duration. The high frequency edge of the fine structure groups slowly drifts towards lower frequency, with rates less than $100 \mathrm{MHz} \mathrm{s}^{-1}$.

Those fine structures were interpreted as a possible signature of chromospheric evaporation process in decimetric 
wavelengths. In this case, the evaporation shock front causes the frequency drift of layer with reduced optical thickness, from where the decimetric radiation can escape. This drift depends upon the evaporation front velocity. Thus, there is a drifting "window" in which the electromagnetic radiation of plasma emission can escape and can be observed. The scenario of the visibility "window" for decimetric emission (1000-2500 MHz) drifting towards lower frequencies is valid at the very beginning of the flare, during the pre-impulsive phase in which the accelerated electron beam is heating the dense plasma. However, we have observed slowly drifting fine structures during the pre-flare phase but also associated with the gradual phase. These results also reported previously by [30] is an evidence that, even in the late phases of some particular flares, the dynamic of the heated plasma into the magnetic configuration permits the escape of the electromagnetic radiation in the decimetric range.

We considered the plasma emission at second harmonic, which has higher possibility to escape from dense layer of the solar atmosphere $\left(10^{9} \mathrm{~cm}^{-3}\right)$ and we have used a density model for the solar chromosphere based on frequency drift rates of type III bursts observations [29]. From the frequency drift rate of the high frequency edge of the fine structures we are able to estimate the front velocity of the evaporation shock in the range of $30-1700 \mathrm{~km} \mathrm{~s}^{-1}$. These velocities are comparable with those computed by Mariska et al. [21] and Karlický [10] and other authors as mentioned in the Introduction, and they agreed with the upper limit of the chromospheric evaporation velocities reported by Fisher et al. [31].

These results permit to interpret those fine structures as a possible signature of the chromospheric evaporation phenomenon in decimetric wavelengths. Complementary investigations of those emissions in other wavelengths are ongoing to confirm the results and will be reported elsewhere.

\section{Acknowledgements}

The BSS Project is supported by INPE, CNPq and FAPESP. F.C.R. Fernandes thanks MCT-CNPq for receiving scholarship (PCI-3849/2002-06). L.C.P. Moraes thanks CNPq for IC scholarship (PIBIC).

\section{References}

[1] F. Droege, Astron. Astrophys. 57, 285 (1977).

[2] M. Guedel and A. O. Benz, Astron. Astrophys. Supp. Series 75, 243 (1988)

[3] M. A. F. Allaart, J. Nieuwkoop, C. Slottje and L. H. Sondaar, Solar Phys. 130, 183 (1990).

[4] H. Isliker and A. O. Benz, Astron. Astrophys. Supp. Series 104, 145 (1994).

[5] R. Moore et al., Report of NASA Skylab Workshop on Solar Flares, Colorado (1980).

[6] A. Kruger and W. Voight, Solar Phys. 161, 393 (1995).

[7] F. C. R. Fernandes, PhD Thesis, INPE-6396-TDI/612, INPE, São José dos Campos, Brazil (1997).
[8] H. S. Sawant, K. R. Subramanian, C. Faria, F. C. R. Fernandes, J. H. A. Sobral, J. R. Cecatto, R. R. Rosa, H. O. Vats, J. A. C. F. Neri, E. M. B. Alonso, F. P. V. Mesquita, V. A. Portezani and A. R. F. Martinon, Solar Phys. 200, 167 (2001).

[9] M. Karlický, F. Farnik and H. Meszarosova, Astron. Astrophys. 395, 677 (2002).

[10] M. Karlický, Astron. Astrophys. 338, 1084 (1998).

[11] M. J. Aschwanden and A. O. Benz, Astrophys. J. 438, 997 (1995).

[12] E. Antonucci, A. H. Gabriel and B. R. Dennis, Astrophys. J. 287, 917 (1984).

[13] A. V. R. Silva, H. Wang, D. E. Gary, N. Nitta and H. Zirin, Astrophys. J. 481, 978 (1997).

[14] P. A. Sturrock, Bulletin of the American Astronomical Society, 5, 28 (1973).

[15] E. Antonucci, B. R. Dennis, A. H. Gabriel and G. M. Simnett, Solar Phys. 96, 129 (1985).

[16] K. Tanaka, Astrophysics and Space Science 118, 101 (1986).

[17] T. Watanabe, Solar Phys. 126, 351 (1990).

[18] E. Antonucci, M. A. Dodero and R. Martin, Astrophys. J. Supp. Series 73, 147 (1990).

[19] L. Teriaca, A. Falchi, G. Cauzzi, R. Falciani, L. A. Smaldone and V. Andretta, Astrophys. J. 588, 596 (2003).

[20] G. Cauzzi, A. Falchi, L. Teriaca, R. Falciani, L. A. Smaldone and V. Andretta, S. Vito Conference Ser., 561 (2002).

[21] J. T. Mariska, A. G. Emslie and P. Li, Astrophys. J. 341, 1067 (1989).

[22] F. C. R. Fernandes, J. R. Cecatto, J. A. C. F. Neri, C. Faria, A. R. F. Martinon, R. R. Rosa,F. P. V. Mesquita, V. A. Portezani, M. C. Andrade, E. M. B. Alonso, H. O. Vats and H. S. Sawant, Boletim da SAB 20, 33 (2000).

[23] H. S. Sawant, K. R. Subramanian, C. Faria, S. Stephany, F. C. R. Fernandes, J. R. Cecatto, R. R. Rosa, V. A. Portezani, F. P. V. Mesquita and E. M. B. Alonso, Astron. Soc. Pacific Conf. Ser. 206, 347 (2000).

[24] F. C. R. Fernandes, J. R.; Cecatto, J. A. C. F. Neri, M. J. B. Silva,R. R. Rosa, C. Faria, M. C. Andrade, E. M. A. Alonso, A. R. F. Martinon, K. M. Dobrowolski, T. M. Barbosa, D. C. Freitas, V. F. Nascimento, A. L. Reis and H. S. Sawant, Boletim da SAB 21, 49 (2001).

[25] F. C. R. Fernandes, Scientific Report INPE-9654-RPQ/740. INPE, São José dos Campos (2003a).

[26] F. C. R. Fernandes, Scientific Report INPE-9653-RPQ/739. INPE, São José dos Campos (2003b).

[27] F. C. R. Fernandes, Scientific Report INPE-9652-RPQ/738. INPE, São José dos Campos (2003c).

[28] F. C. R. Fernandes, Scientific Report INPE-9881-RPQ/742. INPE, São José dos Campos (2003d).

[29] J. L. Melendez, H. S. Sawant, F. C. R. Fernandes and A. O. Benz, A. O., Solar Phys. 187, 77 (1999).

[30] A. Czaykowska, B. De Pontieu, D. Alexander and G. Rank, Astrophys. J. 521, L75 (1999).

[31] G. Fisher, R. C. Canfield and A. N. McClymont, Astrophys. J. 281, L79 (1984). 